\title{
High Definition Wearable Video Communication ${ }^{\star}$
}

\author{
Ulrik Söderström and Haibo Li \\ Digital Media Lab, Dept. Applied Physics and Electronics, \\ Umeå University, \\ SE-90187, Umeå, Sweden \\ \{ulrik.soderstrom, haibo.li\}@tfe.umu.se
}

\begin{abstract}
High definition (HD) video can provide video communication which is as crisp and sharp as face-to-face communication. Wearable video equipment also provide the user with mobility; the freedom to move. HD video requires high bandwidth and yields high encoding and decoding complexity when encoding based on DCT and motion estimation is used. We propose a solution that can drastically lower the bandwidth and complexity for video transmission. Asymmetrical principal component analysis can initially encode $\mathrm{HD}$ video into bitrates which are low considering the type of video $(<300 \mathrm{kbps})$ and after a startup phase the bitrate can be reduced to less than $5 \mathrm{kbps}$. The complexity for encoding and decoding of this video is very low; something that will save battery power for mobile devices. All of this is done only at the cost of lower quality in frame areas which aren't considered semantically important.
\end{abstract}

\section{Introduction}

As much as $65 \%$ of communication between people is determined by non-verbal cues such as facial expressions and body language. Therefore, face-to-face meetings are indeed essential. It is found that face-to-face meetings were more personal and easier to understand than phone or email. It is easy to see that face-to-face meetings are clearer than email since you can get direct feedback; email is not realtime communication. Face-to-face meetings were also seen as more productive and the content easier to remember. But, face-to-face does not need to be in person. Distance communication through video conference equipment is a human-friendly technology that provides the face-to-face communications that people need in order to work together productively, without having to travel. The technology also allows people who work at home or teleworkers to collaborate as if they actually were in the office. Even if there are several benefits with video conferencing it is not very popular. In most cases, video phones have not been a commercial success, but there is a market on the corporate side. Video conferencing with HD resolution can give the impression of face-to-face communication even over networks.

\footnotetext{
^ The wearable video equipment used in this work is constructed by Easyrig AB.
} 
HD video conference essentially can eliminate the distance and make the world connected. On a communication link with HD resolution you can look people in the eye and see whether they follow your argument or not.

Two key expressions for video communication are anywhere and anytime. Anywhere means that communication can occur at any location, regardless of the available network, and anytime means that the communication can occur regardless of the surrounding network traffic or battery power. To achieve this there are several technical challenges:

1. The usual video format for video conference is CIF ( $352 \times 288$ pixels) with a framerate of $15 \mathrm{fps} .1080 \mathrm{i}$ video (1920x1080 pixels) has a framerate of $25 \mathrm{fps}$. Every second there is $\approx 26$ times more data for a $\mathrm{HD}$ resolution video than a CIF video.

2. The bitrate for HD video grows so large that it is impossible to achieve communication over several networks. Even with a high-speed wired connection the bitrate may be too low since communication data is very sensitive to delays.

3. Most of the users want to have high mobility; having the freedom to move while communicating.

A solution for HD video conferencing is to use the H.264 [1, 2] video compression standard. This standard can compress the video to high quality video. There are however two major problems with H.264:

1. The complexity for H.264 coding is quite high. High complexity means high battery consumption; something that is becoming a problem with mobile battery-driven devices. The power consumption is directly related to the complexity so high complexity will increase the power usage.

2. The bitrate for H.264 encoding is very high. The vision of providing video communication anywhere cannot be fulfilled with the bitrates required for H.264. The transmission power is related the bitrate so low bitrate will save battery power.

H.264 encoding cannot provide video neither anywhere or anytime. The question we try to answer in this article is if principal component analysis (PCA) 3] video coding [4, 5] can fulfill the requirements for providing video anywhere and anytime.

The bitrate for PCA video coding can be really low; below $5 \mathrm{kbps}$. The complexity for PCA encoding is linearly dependent on the number of pixels in the frames; when HD resolution is used the complexity will increase and consume power. PCA is extended into asymmetrical PCA (aPCA) which can reduced the complexity for both encoding and decoding [6, 7]. aPCA can encode the video by using only a subset of the pixels while still decoding the entire frame. By combining the pixel subset and full frames it is possible to relieve the decoder of some complexity as well. For PCA and aPCA it is essential that the facial features are positioned on approximately the same pixel positions in all frames so a wearable video equipment is very important for coding based on PCA. 
aPCA enables protection of certain areas within the frame; areas which are important. This area is chosen as the face of the person in the video. We will show how aPCA outperforms encoding with discrete cosine transform (DCT) of the video when it comes to quality for the selected region. The rest of the frame will have poorer reconstruction quality with aPCA compared to DCT encoding. For H.264 video coding it is also possible to protect a specific area by selecting a region of interest (ROI); similarly to aPCA. For encoding of this video the bitrate used for the background is very low and the quality of this area is reduced. So the bitrate for H.264 can be lowered without sacrificing quality for the important area but not to the same low bitrate as aPCA. Video coding based on PCA has the benefit of a much lower complexity for encoding and decoding compared to H.264 and this is a very important factor. The reduced complexity can be achieved at the same time as the bitrate for transmission is reduced. This lowers the power consumption for encoding, transmission and decoding.

\subsection{Intracoded and Intercoded Frames}

H.264 encoding uses transform coding with discrete cosine transform (DCT) and motion estimation through block matching. There are, at least, two different coding types associated with H.264; intracoded and intercoded frames. An intracoded frame is compressed as an image, which it is. Intercoded frames encode the differences from the previous frame. Since frames which are adjacent in time usually share large similarities in appearance it is very efficient to only store one frame and the differences between this frame and the others. Only the first frame in a sequence is encoded through DCT. For the following frames only the changes between the current and first frame is encoded. The number of frames between intracoded frames are called the group of pictures (GOP). A large GOP size means fewer intracoded frames and lower bitrate.

\section{Wearable Video Equipment}

Recording yourself with video usually requires that another person carries the camera or that you use a tripod to place the camera on. When the camera is placed on a tripod the movements that you can make are restricted since the camera cannot move; except for the movements that can be controlled remotely. A wearable video equipment allows the user to move freely and have both hands free for use while the camera follows the movements of the user. The equipment is attached to the back of the person wearing it so the camera films the user from the front. The equipment that we have used is built by the company Easyrig AB and resembles a backpack; it is worn on the back (Figure 11). It consists of a backpack, an aluminium arm and a mounting for a camera at the tip of the arm.

\section{High Definition (HD) Video}

High-definition (HD) video refers to a video system with a resolution higher than regular standard-definition video used in TV broadcasts and DVD-movies. The 


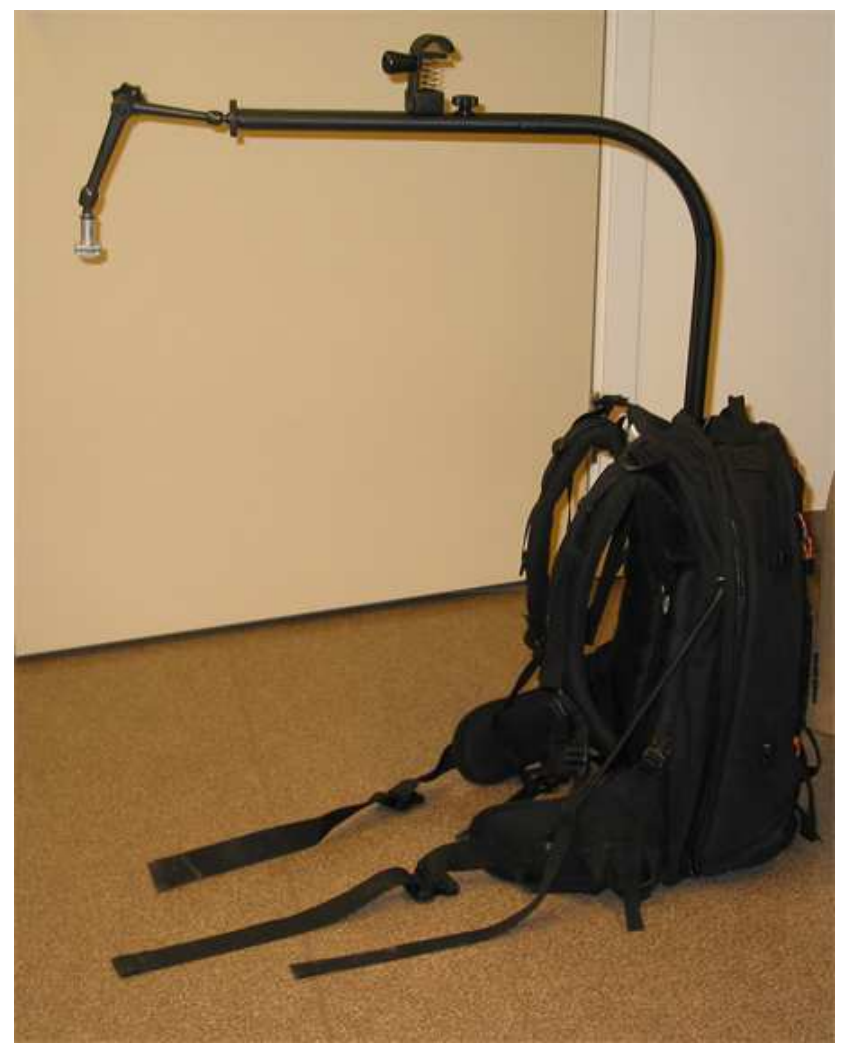

Fig. 1. Wearable video equipment

display resolutions for HD video are called 720p (1280x720), 1080i and 1080p (both 1929x1080). i stands for interlaced and p for progressive. Each interlaced frame is divided into two parts where each part only contains half the lines of the frame. The two parts contain either odd or even lines and when they are displayed the human eye perceives that the entire frame is updated. TVtransmissions that have HD resolution use either 720p or 1080i; in Sweden it is mostly 1080i. The video that we use as HD video has a resolution of 1440x1080 (HD anamorphic). It is originally recorded as interlaced video with 50 interlace fields per second but it is transformed into progressive video with 25 frames per second.

\section{Wearable Video Communication}

Wearable video communication enables the user to move freely; the users mobility is largely increased compared to regular video communication. 
The wearable equipment is described in section 2 and video recorded with this equipment is efficiently encoded with principal component analysis (PCA). PCA [3] is a common tool for extracting compact model of faces [8]. A model of a persons facial mimic is called personal face space, facial mimic space or personal mimic space [9, 10]. This space contain the face of the same person but with several different facial expressions. This model can be used to encode video and images of human faces [11, 12, or the head and shoulders of a person 4, 13. to extremely low bitrates.

A space that contains the facial mimic is called Eigenspace $\Phi$ and it is constructed as

$$
\phi_{i}=\sum_{j} b_{i j}\left(\mathbf{I}-\mathbf{I}_{\underline{0}}\right)
$$

where $\mathbf{I}$ are the original frames and $\mathbf{I}_{\underline{0}}$ is the mean of all video frames. $b_{i j}$ are the Eigenvectors from the the covariance matrix $\left(\mathbf{I}-\mathbf{I}_{0}\right)^{T}\left(\mathbf{I}-\mathbf{I}_{0}\right)$. The Eigenspace $\Phi$ consists of the principal components $\phi_{j}\left(\Phi=\left\{\phi_{j} \bar{\phi}_{j+1} \ldots \phi_{N}\right\}\right)$. Encoding of a video frame is done through projection of the video frame onto the Eigenspace $\Phi$.

$$
\alpha_{j}=\phi_{j}\left(\mathbf{I}-\mathbf{I}_{\underline{0}}\right)^{T}
$$

where $\left\{\alpha_{j}\right\}$ are projection coefficients for the encoded video frame. The video frame is decoded by multiplying the projection coefficients $\left\{\alpha_{j}\right\}$ with the Eigenspace $\Phi$.

$$
\hat{\mathbf{I}}=\mathbf{I}_{\underline{0}}+\sum_{j=1}^{M} \alpha_{j} \phi_{j}
$$

where $M$ is a selected number of principal components used for reconstruction $(M<N)$. The extent of the error incurred by using fewer components $(M)$ than possible $(N)$ is examined in [5]. With asymmetrical PCA (aPCA) one part of the image can be to encode the video and a different part can be decoded [6. 7. Asymmetrical PCA uses pseudo principal components; information where not the entire frame is a principal component. Parts of the video frames are considered to be important; they are regarded as foreground $\mathbf{I}^{f}$. The Eigenspace for the foreground $\Phi^{f}$ is constructed according to the following formula:

$$
\phi_{j}^{f}=\sum_{j} b_{i j}^{f}\left(\mathbf{I}^{f}-\mathbf{I}_{\underline{0}}^{f}\right)
$$

where $b_{i j}^{f}$ are the Eigenvectors from the the covariance matrix $\left(\mathbf{I}^{f}-\mathbf{I}_{\underline{0}}^{f}\right)^{T}\left(\mathbf{I}^{f}-\mathbf{I}_{\underline{0}}^{f}\right)$ and $\mathbf{I}_{\underline{0}}^{f}$ is the mean of the foreground.

A space which is spanned by components where only the foreground is orthogonal can be created. The components spanning this space are called pseudo principal components and this space has the same size as a full frame:

$$
\phi_{j}^{p}=\sum_{j} b_{i j}^{f}\left(\mathbf{I}-\mathbf{I}_{\underline{0}}\right)
$$


Encoding is performed using only the foreground:

$$
\alpha_{j}^{f}=\left(\mathbf{I}^{f}-\mathbf{I}_{\underline{0}}^{f}\right)^{T} \phi_{j}^{f}
$$

where $\left\{\alpha_{j}^{f}\right\}$ are coefficients extracted using information from the foreground $\mathbf{I}^{f}$. By combining the pseudo principal components $\Phi^{p}$ and the coefficients $\left\{\alpha_{j}^{f}\right\}$ full frame video can be reconstructed.

$$
\hat{\mathbf{I}}^{p}=\mathbf{I}_{\underline{0}}+\sum_{j=1}^{M} \alpha_{j}^{f} \phi_{j}^{p}
$$

where $M$ is the selected number of pseudo components used for reconstruction.

By combining the two Eigenspaces $\Phi^{p}$ and $\Phi^{f}$ we can reconstruct frames with full frame size and reduce the complexity for reconstruction. Only a few principal components of $\Phi^{p}$ are used to reconstruct the entire frame. More principal components from $\Phi^{f}$ is used to add details to the foreground.

$$
\hat{\mathbf{I}}^{\prime}=\mathbf{I}_{\underline{0}}+\sum_{j=1}^{P} \alpha_{j} \phi_{j}^{p}+\sum_{j=P+1}^{M} \alpha_{j} \phi_{j}^{f}
$$

The result is reconstructed frames with slightly lower quality for the background but with the same quality for the foreground $\mathbf{I}^{f}$ as if only $\Phi^{p}$ was used for reconstruction. By adjusting the parameter $\mathrm{P}$ it is possible to control the bitrate needed for transmission of Eigenimages. Since P decides how many Eigenimages of $\Phi^{p}$ that are used for decoding it also decides how many Eigenimages of $\Phi^{p}$ that needs to be transmitted to the decoder. $\Phi^{f}$ has a much smaller spatial size than $\Phi^{p}$ and transmission of an Eigenimage from $\Phi^{f}$ requires fewer bits than transmission of an Eigenimage from $\Phi^{p}$.

A third space $\Phi^{p^{b g}}$ which contain only the background and not the entire frame is easily created. This is a space with pseudo principal components; this space is exactly the same as $\Phi^{p}$ without information from the foreground $\mathbf{I}^{f}$.

$$
\phi_{j}^{p^{b g}}=\sum_{j} b_{i j}^{f}\left(\mathbf{I}^{b g}-\mathbf{I}_{\underline{0}}^{b g}\right)
$$

where $\mathbf{I}^{b g}$ is frame $\mathbf{I}$ minus the pixels from the foreground $\mathbf{I}^{f}$. This space is combined with the space from the foreground to create reconstructed frames.

$$
\hat{\mathbf{I}}^{\prime}=\mathbf{I}_{\underline{0}}+\sum_{j=1}^{M} \alpha_{j} \phi_{j}^{f}+\sum_{j=1}^{P} \alpha_{j} \phi_{j}^{b g}
$$

The result is exactly the same as for Eq. (8); high foreground quality, lower background quality, reduced decoding complexity and reduced bitrate for Eigenspace transmission.

When both the encoder and decoder have access to the model of facial mimic the bitrate needed for this video is extremely low $(<5 \mathrm{kbps})$. If the model needs 
to be transmitted between the encoder and decoder almost the entire bitrate need consists of bits for model transmission.

The complexity for encoding through PCA is linearly dependent on the spatial resolution. The complexity for PCA encoding is dependent on the number of pixels $K$ in the frame. This complexity can be reduced for aPCA since $K$ is a much smaller value for aPCA compared to PCA.

\section{HD Video with H.264}

As a comparison of HD video encoded with aPCA we encode the video sequence with H.264 as well. We use the same software for encoding of the entire video as for encoding of the Eigenimages; but we also enable motion estimation. The entire video is encoded with H.264 with a target bitrate of $300 \mathrm{kbps}$. To get this bitrate we encode the video with a quantization step of 29 . We compare the quality of the foreground and background separately since they have different qualities when aPCA is used. With standard H.264 encoding the quality for the background and foreground are approximately equal.

The complexity for H.264 encoding is linearly dependent on the frame size. Most of the complexity for H.264 encoding comes from motion estimation through block matching. The blocks has to be matched for several positions and the blocks can move both in horizontal and vertical direction. The complexity for H.264 encoding is dependent on $K$ and the displacement $D$ in square $\left(D^{2}\right)$. When the resolution is increased the number of displacements are increased. Imagine a line in a video with CIF resolution. This line will consist of a number of pixels, e.g., 5. If the same line is described in HD resolution the number of pixels in the line will increase to almost 19. If the same movement between frames is used in CIF and HD the displacement in pixels is much higher for HD video. When motion estimation is used for H.264 video the complexity grows high because of $D^{2}$. So even if the complexity is only linearly dependent on the number of pixels $K$ the complexity grows more faster than linearly for high resolution video.

\section{HD Video at Low Bitrates}

aPCA can be utilized by the decoder to decode parts of the same frame with different spatial resolution. Since the same part of the frame $\mathbf{I}^{f}$ is used for encoding in both cases, the decoder can choose to decode either $\mathbf{I}^{f}$ or the entire frame I. The decoded video can also be a combination of $\mathbf{I}^{f}$ and $\mathbf{I}$. This is described in detail in [7]. How $\Phi^{f}$ and $\Phi^{p}$ are combined can be selected by a number of parameters, such as quality, complexity or bitrate. In this article we will focus on bitrate and complexity.

\subsection{Bitrate Calculations}

The bitrate that we select as a target for video transmission is $300 \mathrm{kbps}$. The video needs to be transmitted below this bitrate at all times. The frame size 
for the video is $1440 \times 1080(\mathbf{I})$. The foreground in this video is $432 \times 704\left(\mathbf{I}^{f}\right)$ (Figure 2). After YUV 4:1:1 compression the number of pixels in the foreground is 456192. The entire frame $\mathbf{I}$ consists of 2332800 pixels and the frame area which is not foreground is 1876608 pixels. The video has a framerate of $25 \mathrm{fps}$ but this has only slight impact on the bitrate for aPCA since each frame is encoded to a few coefficients. The bitrate for these coefficients is easily kept below $5 \mathrm{kbps}$. Audio is an important part of communication but we will not discuss this in our work. There are several codecs that can provide audio with good quality at a bitrate which can be used. We use $300 \mathrm{kbps}$ for transmission of the Eigenimages $\left(\Phi^{p}\right.$ and $\left.\Phi^{f}\right)$ and the coefficients $\left\{\alpha_{j}^{f}\right\}$ between sender and receiver.

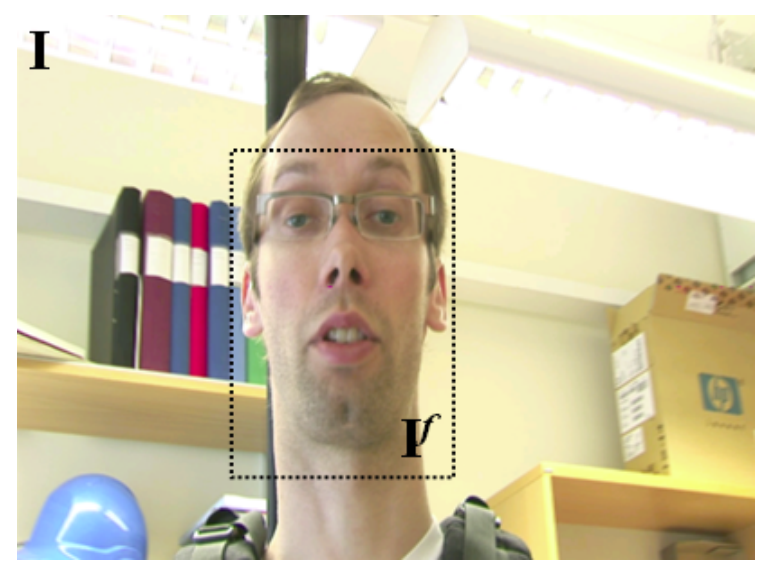

Fig. 2. Frame with the foreground shown

Transmission of the Eigenimages $\phi_{j}$ means transmission of images. The Eigenimages have too large size $\approx 7,5 \mathrm{MB}(1440 \times 1080$ resolution minus the foreground) to be transmitted without compression. Since they are images we could use image compression but the images share large similarities in appearance; the facial mimic is independent between the images but it is the same face with similar background. Globally the images are not only uncorrelated but also independent and doesn't share any similarities. Image or video compression based on DCT divides the frames into blocks and encodes each block individually. Even though the frames are independent globally it is possible to find local similarities so to consider the images as a sequence will provide higher compression. We want to remove the complexity associated with motion estimation and only encode the images through DCT.

We use the H.264 video compression without any motion estimation; this encoding uses both intracoding and intercoding. The first image is intracoded and the subsequent images are intercoded but without motion estimation. The mean image is only one image so we will use the JPEG [14] standard for compression of it. The mean image is in fact compressed in the same manner as in $[5]$. 
To make the compression more efficient we first use quantization of the images. In our previous article we discussed the usage of pdf-optimized or uniform quantization extensively and came to the conclusion that it is sufficient to use uniform quantization [5]. So, in this work we will use uniform quantization. In our previous work we also examined the effect of high compression and loss of orthogonality between the Eigenimages. To retain high visual quality on the reconstructed frames we will not use so high compression that the loss of orthogonality becomes an important factor. The compression is achieved through the following steps:

- Quantization of the Eigenimages. $\Phi^{Q}=\mathrm{Q}(\Phi)$

- The Eigenimages are compressed. $\Phi^{C o m p}=\mathrm{C}\left(\Phi^{Q}\right)$

- Reconstruction of the Eigenimages from compressed video. $\hat{\Phi}^{Q}=\mathrm{C}^{\prime}\left(\Phi^{C o m p}\right)$

- Inverse quantization mapping of the quantization values with the reconstruction values. $\hat{\Phi}=\mathrm{Q}^{\prime}\left(\hat{\Phi}^{Q}\right)$

The mean image $\mathbf{I}_{0}$ is compressed in a similar way but we use JPEG compression instead of H.264. We have $295 \mathrm{kbps}$ for Eigenimage transmission and this is equal to $\approx 60 \mathrm{kB}$. The foreground $\mathbf{I}^{f}$ have a size of $\approx 1,8 \mathrm{MB}$ when it is uncompressed. It is possible to choose from a wide range of compression grades when it comes to encoding with DCT. We select a compression ratio based on reconstruction quality that the Eigenimages provides and the bitrate which is needed for transmission of the video; the compression is chosen by the following criteria.

- A compression ratio that allow the use of a bitrate below our needs.

- A compression ratio that provide sufficiently high reconstruction of video when compressed Eigenimages are used for encoding and decoding of video.

The first criteria decides how fast the Eigenimages can be transmitted; e.g., how fast high quality video can be decoded. The second criteria decides the quality of reconstructed video.

\section{7 aPCA Decoding of HD Video}

The face is the most important information in the video so Eigenimages $\phi_{j}^{f}$ for the foreground $\mathbf{I}^{f}$ is transmitted first. The bitrate for the compressed Eigenimages $\phi^{f^{\text {Comp }}}$ is $13 \mathrm{kbps}$ but the bitrate for the first Eigenimage is higher since it is intracoded. The background is larger in spatial size so the bitrate for this is $42 \mathrm{kbps}$. Transmission of 10 Eigenimages for the foreground $\phi^{C o m p^{f}}, 1$ pseudo Eigenimage for the background $\phi^{C o m p} p^{p}$ plus the mean for both areas can be done within 1 second. After $\approx 220 \mathrm{~ms}$ the first Eigenimage and the mean for the foreground is available and decoding of the video can start. All the other Eigenimages are intercoded and a new image arrives every 34 th ms. After $\approx$ $520 \mathrm{~ms}$ the decoder has 10 Eigenimages for the foreground. The mean and the first Eigenimage for the background needs $\approx 460 \mathrm{~ms}$ for transmission and a new 


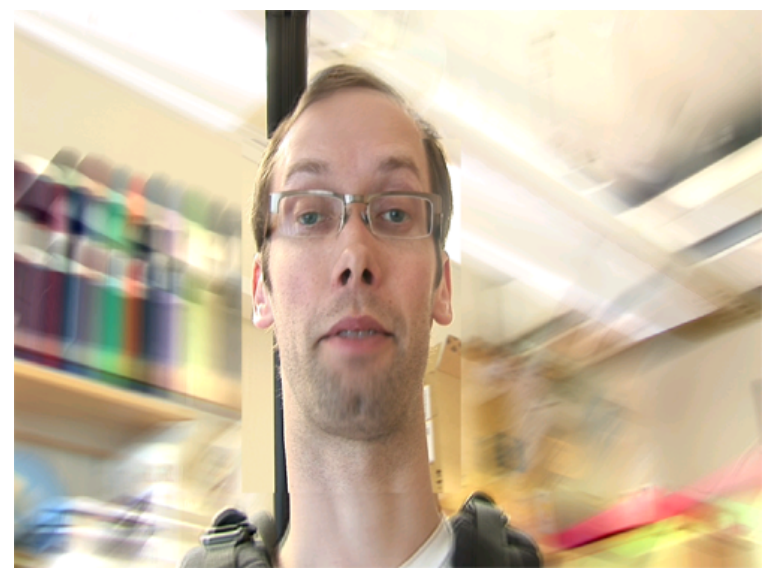

Fig. 3. Frame reconstructed with aPCA $\left(25 \phi_{j}^{f}\right.$ and $5 \phi_{j}^{p}$ are used $)$

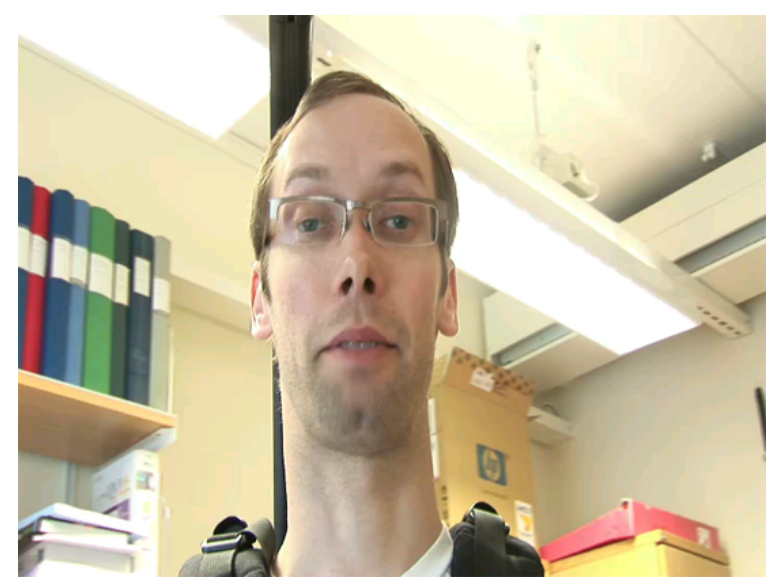

Fig. 4. Frame encoded with H.264

Eigenimage for the background can be transmitted every 87 th $\mathrm{ms}$. The quality of the reconstructed video is increased as more Eigenimages arrive. There doesn't have to be a stop to the quality improvement; more and more Eigenimages can be transmitted. But when all Eigenimages that the decoder wants to use for decoding has arrived only the coefficients needs to be transmitted so the bitrate is then below $5 \mathrm{kbps}$. The Eigenimages can also be updated; something we examined in [5]. The Eigenspace may need to be updated because of loss of alignment between the model and the new video frames.

The average results measured in psnr for the video sequences are shown in table 1 and table 2. Table 1 show the results for the foreground and 2 show the results for the background. The results in the tables are for full decoding 
Table 1. Reconstruction quality for the foreground

\begin{tabular}{|c|c|c|c|}
\cline { 2 - 4 } \multicolumn{1}{c|}{} & \multicolumn{3}{c|}{ Rec. qual. PSNR [dB] } \\
\cline { 2 - 4 } \multicolumn{1}{c|}{} & Y & U & V \\
\hline H.264 & 36.4 & 36.5 & 36.5 \\
\hline aPCA & 44.2 & 44.3 & 44.3 \\
\hline
\end{tabular}

Table 2. Reconstruction quality for the background

\begin{tabular}{|c|c|c|c|}
\cline { 2 - 4 } \multicolumn{1}{c|}{} & \multicolumn{3}{c|}{ Rec. qual. PSNR [dB] } \\
\cline { 2 - 4 } \multicolumn{1}{c|}{} & Y & U & V \\
\hline H.264 & 36.3 & 36.5 & 36.6 \\
\hline aPCA & 29.6 & 29.7 & 29.7 \\
\hline
\end{tabular}

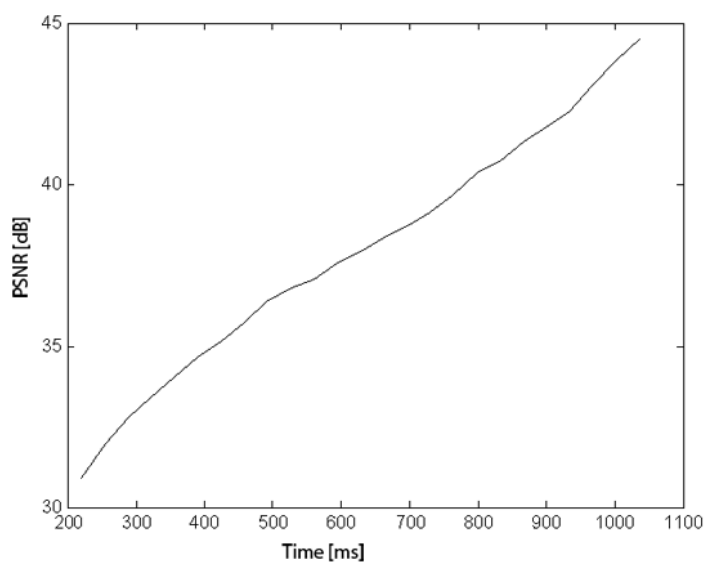

Fig. 5. Foreground quality (Y-channel) over time

quality $\left(25 \phi_{j}^{f}\right.$ and $\left.5 \phi_{j}^{p}\right)$. Figure 5 show how the foreground quality of the Ychannel is increased over time for aPCA. Figure 6] show the same progress for the background. An example of a frame reconstructed with aPCA is shown in figure 3. A reconstructed frame from H.264 encoding is shown in figure 4 .

As it can be seen from the tables and the figures the background quality is always lower for aPCA compared with H.264. This will not change even if all Eigenimages are used for reconstruction; the background is always blurred. The exception is when the background is homogenous but the quality of this background with H.264 encoding is also very good.

The foreground quality for aPCA is better than H.264 already when 10 Eigenimages (after $\approx 1$ second) are used for reconstruction and just improves after that. 


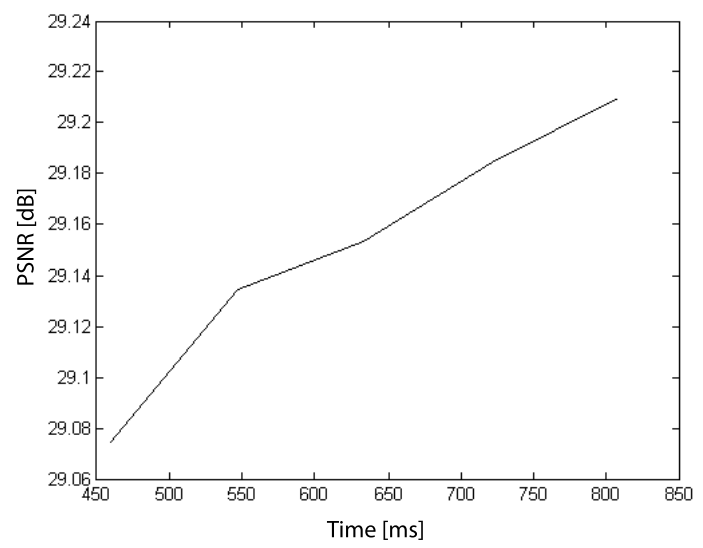

Fig. 6. Background quality (Y-channel) over time

That the quality doesn't increase linearly depends on the fact that the Eigenimages that are added to reconstruction have different mimics. The most important mimic is the first so it should improve the quality the most and the subsequent ones should improve the quality less and less. But the 5th expression may improve some frames with really bad reconstruction quality and thus increase the quality more than the 1st Eigenimage. It may also improve the mimic for several frames; the most important mimic can be visible in fewer frames than another mimic which is not as important based on the variance.

\section{Discussion}

The use of aPCA for compression for video with $\mathrm{HD}$ resolution can reduce the bitrate for transmission vastly after an initial transmission of Eigenimages. The available bitrate can also be used to improve the reconstruction quality further. A drawback with any implementation based on PCA is that it is not possible to reconstruct a changing background with high quality; it will always be blurred due to motion.

The complexity for both encoding and decoding is reduced vastly when aPCA is used compared to DCT encoding with motion estimation. This can be an extremely important factor since the power consumption is reduced and any device that is driven by batteries will have longer operating time. Since the bitrate also can be reduced the devices can save power on lower transmission costs as well.

Initially there are no Eigenimages available at the decoder side and no video can be displayed. This initial delay in video communication cannot be dealt with by buffering if the video is used in online communication such as a video telephone conversation. This shouldn't have to be a problem for video conference 
applications since you usually don't start communicating immediately. And a second is enough time to wait for good quality video.

There are possibilities of combining PCA or aPCA with DCT encoding such as H.264 and this will be a hybrid codec. For an initial period the frames can be encoded with H.264 and transmitted between the encoder and decoder. The fames are available at both the encoder and decoder so they can both perform PCA for the images and produce the same Eigenimages. All other frames can then be encoded with the Eigenimages to very low bitrates with low encoding and decoding complexity.

\section{References}

[1] Schäfer, R., et al.: The emerging h.264 avc standard. EBU Technical Review 293 (2003)

[2] Wiegand, T., Sullivan, G.J., Bjontegaard, G., Luthra, A.: Overview of the h.264/avc video coding standard. IEEE Trans. Circuits Syst. Video Technol. 13(7), 560-576 (2003)

[3] Jolliffe, I.: Principal Component Analysis. Springer, New York (1986)

[4] Söderström, U., Li, H.: Full-frame video coding for facial video sequences based on principal component analysis. In: Proceedings of Irish Machine Vision and Image Processing Conference 2005 (IMVIP 2005), August 30-31, 2005, pp. 25-32 (2005), www.medialab.tfe.umu.se

[5] Söderström, U., Li, H.: Representation bound for human facial mimic with the aid of principal component analysis. EURASIP Journal of Image and Video Processing, special issue on Facial Image Processing (2007)

[6] Söderström, U., Li, H.: Asymmetrical principal component analysis for video coding. Electronics letters 44(4), 276-277 (2008)

[7] Söderström, U., Li, H.: Asymmetrical principal component analysis for efficient coding of facial video sequences (2008)

[8] Turk, M., Pentland, A.: Eigenfaces for recognition. Journal of Cognitive Neuroscience 3, 71-86 (1991)

[9] Ohba, K., Clary, G., Tsukada, T., Kotoku, T., Tanie, K.: Facial expression communication with fes. In: International conference on Pattern Recognition, pp. 1378-1378 (1998)

[10] Ohba, K., Tsukada, T., Kotoku, T., Tanie, K.: Facial expression space for smooth tele-communications. In: FG 1998: Proceedings of the 3rd International Conference on Face \& Gesture Recognition, p. 378 (1998)

[11] Torres, L., Prado, D.: A proposal for high compression of faces in video sequences using adaptive eigenspaces. In: 2002 International Conference on Image Processing, 2002. Proceedings, vol. 1, pp. I-189- I-192 (2002)

[12] Torres, L., Delp, E.: New trends in image and video compression. In: Proceedings of the European Signal Processing Conference (EUSIPCO), Tampere, Finland, September 5-8 (2000)

[13] Söderström, U., Li, H.: Eigenspace compression for very low bitrate transmission of facial video. In: IASTED International conference on Signal Processing, Pattern Recognition and Application (SPPRA) (2007)

[14] Wallace, G.K.: The jpeg still picture compression standard. Communications of the ACM 34(4), 30-44 (1991) 